\title{
Management of inferior vena cava thrombosis after blunt liver injury
}

\author{
Kyung-Yun Kim, Byung-Jun So, and Dong-Eun Park \\ Department of Surgery, Wonkwang University School of Medicine, Iksan, Korea
}

\begin{abstract}
Inferior vena cava (IVC) thrombosis after traumatic liver injury is an extremely rare condition, and only 12 cases have been reported in the English literature since 1911. We report a case of a 26-year-old man who presented with IVC thrombosis after blunt liver injury. IVC thrombosis was incidentally detected by computed tomography 15 days after conservative management of blunt liver injury. The patient denied any symptoms of thrombophlebitis and did not have any evidence of hypercoagulable state. We placed an IVC filter via the right jugular vein and started the anticoagulation treatment. The patient recovered successfully without operative treatment and IVC thrombosis disappeared completely two months later. We suggest that that the possibility of IVC thrombosis should be considered in patients with a large hematoma of the liver, which may cause compression of the IVC. (Korean J Hepatobiliary Pancreat Surg 2014;18:97-100)
\end{abstract}

Key Words: Liver injury, Hematoma, Inferior vena cava, Thrombosis

\section{INTRODUCTION}

Inferior vena cava (IVC) thrombosis after hepatic trauma is an extremely rare condition, and only 12 cases have been reported in the English literature since $1911^{1-8}$ Various mechanisms for the origin of this condition have been suggested; but there is no well-established evidence and treatment.

We herein report a case of IVC thrombosis after blunt liver injury that was managed by IVC filter insertion and anticoagulation therapy.

\section{CASE}

A 26-year-old man was taken to the emergency room of a University Hospital after high- speed motor vehicle collision. The patient complained of bilateral knee pain and right upper quadrant abdominal pain. On arrival, the patient was alert (Glasgow coma scale score of 14) and vital signs were as follows: the patient was normothermic, heart rate was 70 beats/min, respiratory rate was $20 / \mathrm{min}$, and blood pressure was $105 / 60 \mathrm{mmHg}$. Physical examina- tion was unremarkable and the findings of the initial blood tests were as follows: hemoglobin $15.0 \mathrm{~g} / \mathrm{dl}$, white blood cell count $8,130 / \mathrm{mm}^{3}$, platelet count $192,000 / \mathrm{mm}^{3}$, aspartate transaminase $491 \mathrm{IU} / \mathrm{L}$, alanine transaminase 495 IU/L, amylase $62 \mathrm{IU} / \mathrm{L}$, and lipase $82 \mathrm{IU} / \mathrm{L}$. Routine trauma series X-rays were normal except for multiple fractures of the right ribs. However, abdominal computed tomography (CT) showed hepatic laceration with parenchymal hematoma in the segments $4,5,7$, and 8 , and moderate amount of intra-abdominal fluid collection (Fig. 1).

Because the patient was hemodynamically stable and he did not have any other visceral organ injuries, he was managed conservatively in the intensive care unit. On follow-up day 2 , there were no changes in the vital signs and there was no decrease in hemoglobin and hematocrit levels. As the patient also did not have any specific symptoms, we continued the conservative treatment. The follow-up abdominal CT on day 5 showed a slight decrease in the size of parenchymal hematoma and intra-abdominal fluid collection (Fig. 2). At this time point, he could walk by himself and could be given a regular diet.

Received: July 17, 2014; Revised: August 14, 2014; Accepted: August 20, 2014

Corresponding author: Dong-Eun Park

Department of Surgery, Wonkwang University School of Medicine, 344-2, Shinyoung-dong, Iksan 570-711, Korea

Tel: +82-63-859-1490, Fax: +82-63-855-2386, E-mail: knife@wonkwang.ac.kr

This paper was supplied by Wonkwang University 2012. 

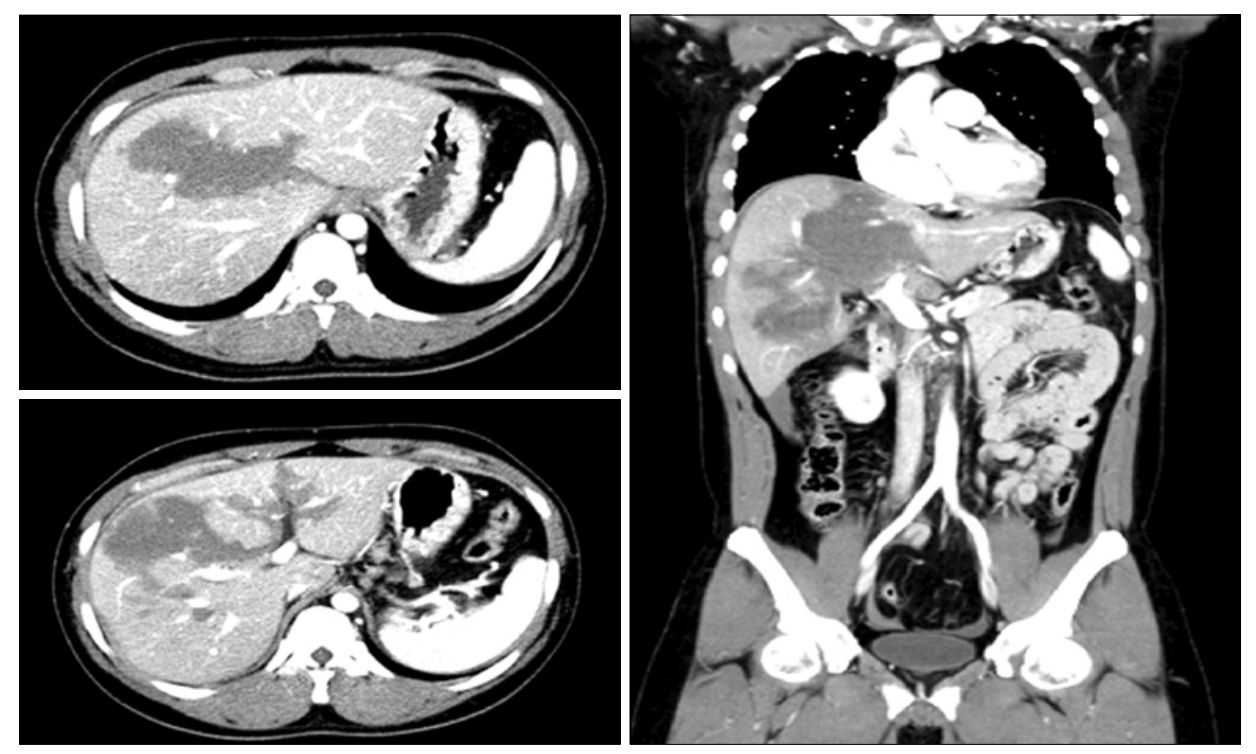

Fig. 1. Computed tomography scan on admission showing multiple liver lacerations.
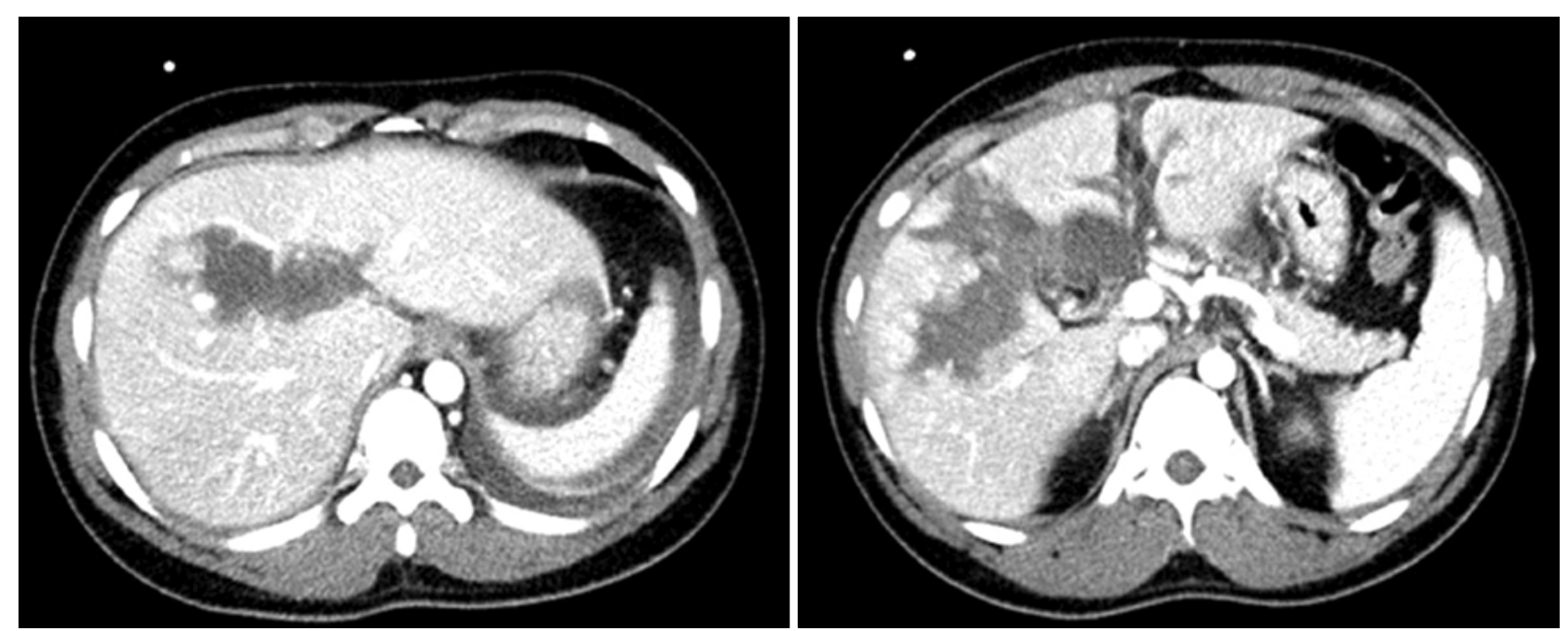

Fig. 2. Follow-up abdominal CT on the follow-up day 5 showing a slight decrease in the size of parenchymal hematoma.

A repeat abdominal $\mathrm{CT}$ on day 15 demonstrated considerable improvement in hepatic laceration and hematoma, but a large thrombus in the IVC (about $10 \mathrm{~cm}$ in length, from L1 to L5 spines) was detected (Fig. 3). The patient did not show any symptoms and signs of thrombophlebitis such as bilateral leg edema, skin color changes, and pain except for vague nonspecific upper abdominal pain. For evaluation of any hypercoagulable state, the specific blood test was performed, but no evidence of hypercoagulability was obtained.

One day later, an IVC filter was placed in the suprarenal IVC via the right jugular vein to protect against the risk of pulmonary embolism (Fig. 4). Localized intra- thrombus lytic therapy was considered; but it was not performed due to concerns with respect to the underlying liver laceration and hematoma. Instead, we started oral anticoagulation therapy and performed close observation of bleeding, but no sign of bleeding was detected. The patient was discharged from hospital at 1 month after admission. On follow-up abdominal CT performed 2 months later, IVC thrombus had disappeared completely (Fig. 5).

\section{DISCUSSION}

IVC thrombosis after hepatic trauma is an extremely rare condition. Since it is a rare entity, the definitive 

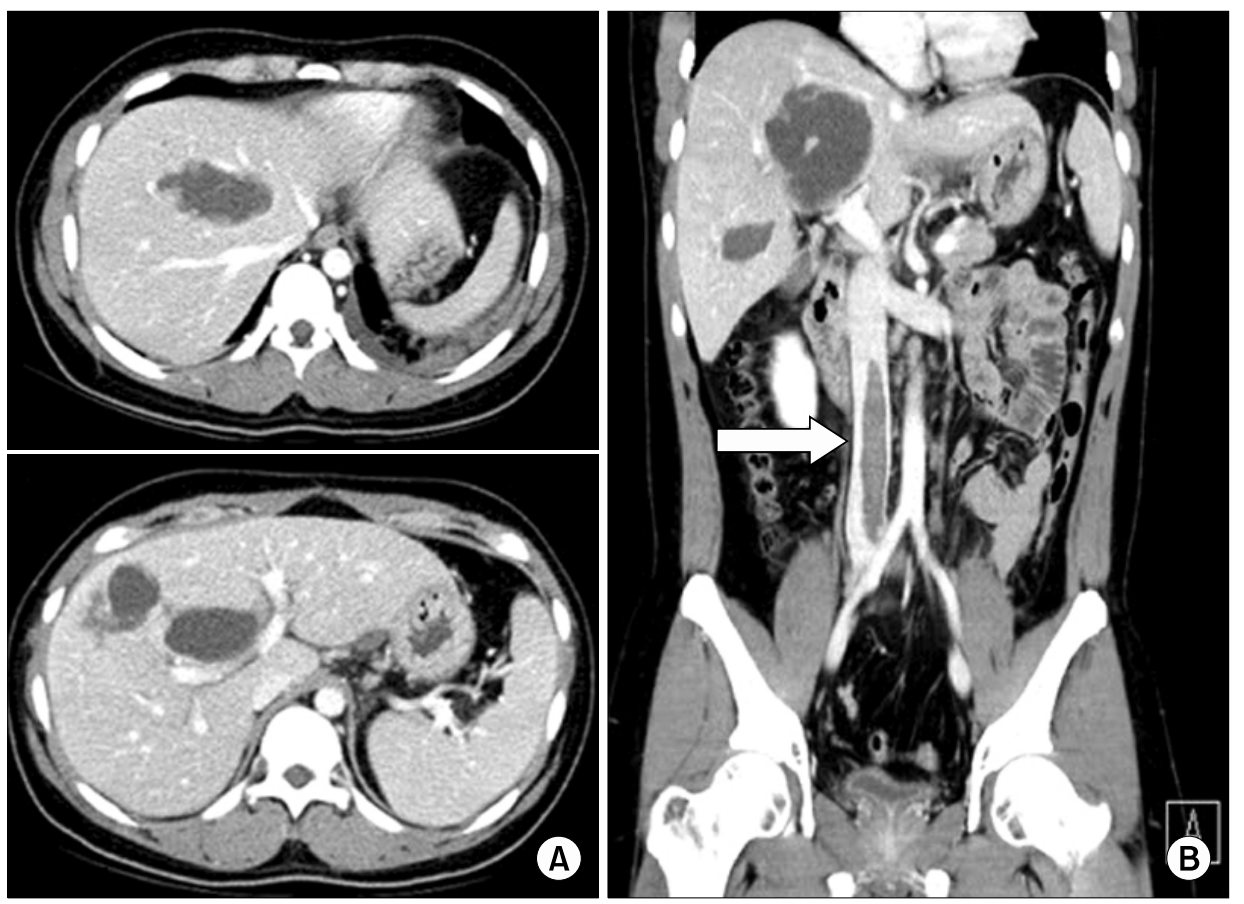

Fig. 3. A repeat abdominal CT on the follow-up day 15 showing significant improvement in hepatic laceration (A) and a large free-floating thrombus in the IVC (white arrow) (B).

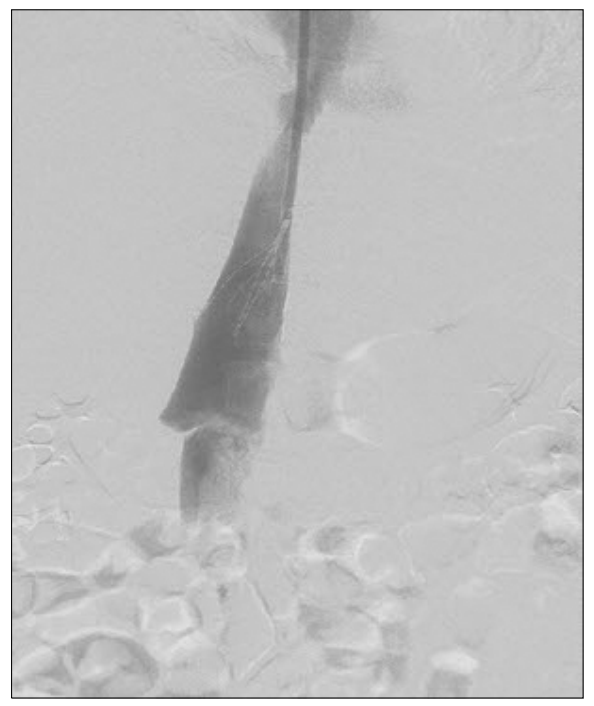

Fig. 4. IVC filter insertion via the right jugular vein.

mechanism of thrombosis and its treatment have not been well elucidated. Nonetheless, various mechanisms have been advocated for IVC thrombosis after hepatic trauma: hepatic vein thrombosis, which extends into the IVC, endothelial injury of the caval wall, caval flow stasis caused by the compression with a pericaval or a retroperitoneal hematoma, and/or hypercoagulable and hypofibrinolytic state after major trauma.

IVC thrombosis has been attributed to transmural laceration of the vena cava secondary to crushing forces, with

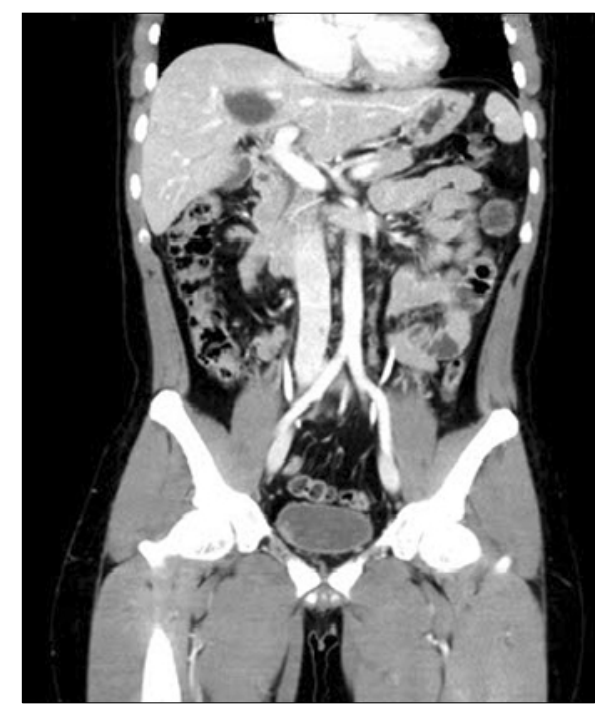

Fig. 5. A vascular CT on the follow-up day 60 showing disappearance of IVC thrombosis and a marked decrease in the liquefied hematoma of the liver.

formation of a pericaval or a retroperitoneal hematoma that compresses and narrows the vena cava to such an extent that venous stasis develops. This stasis leads to distal thrombophlebitis and then antegrade caval thrombosis. ${ }^{2}$ In cases where a retroperitoneal or a pericaval hematoma is not evident, different mechanisms of traumatic thrombosis are involved. Endothelial injury of the venous wall secondary to compression or shear forces leads directly to caval 
mural thrombosis resulting in occlusion. ${ }^{2}$ Hepatic parenchymal injury may cause hepatic vein thrombosis that eventually extends into the IVC; however, this mechanism is most likely to be present in cases with the BuddChiari syndrome. Hypercoagulability associated with suppression of fibrinolysis is a normal physiologic response after trauma. ${ }^{3}$ Alterations in the fibrinolytic system are concurrent with this hypercoagulability. After the initial hyperfibrinolytic state, the overall fibrinolytic activity is strikingly reduced. Based on the above-mentioned mechanisms, hypercoagulability is likely to be the triggering factor for the development of IVC thrombosis.

In the present case, it was difficult to identify the cause of IVC thrombosis because the CT scan did not show a retroperitoneal hematoma around the IVC or a thrombus in the hepatic vein causing caval flow stasis. But, it showed huge hepatic parenchymal hemorrhage extending into the retrohepatic portion of the IVC and injury to the caval wall.

The clinical manifestations of IVC thrombosis after hepatic trauma are unclear and it unusually presents as vague, nonspecific abdominal and lower back pain. ${ }^{4}$ The symptoms and signs of hepatic trauma are also nonspecific unless there are other associated injuries. In the present case, there were no distinctive symptoms and signs in spite of a large thrombus in the IVC. Therefore, regular interval follow-up CT can be the most valuable diagnostic tool for detecting IVC thrombosis.

Hamamoto et al. advocated that massive IVC thrombus prevents the venous drainage from the hepatic veins, resulting in progressive liver congestion, failure, and ultimately death. ${ }^{1}$ Especially, the condition with massive extension of IVC thrombus into the suprahepatic level such as Budd-Chiari syndrome is more dangerous. They suggested that it is important to remove the IVC thrombus and decompress the congested liver as early as possible before irreversible liver failure occurs. In contrast, Agos et al. suggested that timely insertion of an IVC filter for pulmonary embolism prophylaxis is necessary. ${ }^{5}$

In our opinion, a massive thrombus extending to the suprahepatic level needs emergency thrombectomy; otherwise non-operative treatment such as IVC filter and/or anticoagulation regimen can be chosen depending on the situation. In the present case, there was a large floating thrombus in the IVC; but it did not extend to the suprahepatic level.

In conclusion, we propose that the possibility of IVC thrombosis should be considered in patients with severe hepatic trauma above grade III (AAST liver injury scale) and regular follow-up CT can be an important diagnostic method to make an early diagnosis of IVC thrombosis. The IVC filter with/without anticoagulation treatment is likely to be the best initial option for prophylaxis of pulmonary embolism if it has not extended to the suprahepatic level such as in Budd-Chiari syndrome.

\section{REFERENCES}

1. Hamamoto M, Kobayashi T, Kodama H, Nakamitsu A, Sasaki M, Kuroo Y. Thrombectomy under cardiopulmonary bypass for inferior vena cava thrombosis induced by liver injury. Ann Vasc Dis 2013;6:751-755.

2. Campbell DN, Liechty RD, Rutherford RB. Traumatic thrombosis of the inferior vena cava. J Trauma 1981;21:413-415.

3. Kimoto T, Kohno H, Uchida M, Yamanoi A, Yamamoto A, Nagasue $\mathrm{N}$, et al. Inferior vena caval thrombosis after traumatic liver injury. HPB Surg 1998;11:111-116.

4. Nagy KK, Duarte B. Post-traumatic inferior vena caval thrombosis: case report. J Trauma 1990;30:218-221.

5. Boggi U, Vistoli F, Del Chiaro M, Signori S, Sgambelluri F, Roncella M, et al. Extracorporeal repair and liver autotransplantation after total avulsion of hepatic veins and retrohepatic inferior vena cava injury secondary to blunt abdominal trauma. J Trauma 2006;60:405-406.

6. Patel NH, Bradshaw B, Meissner MH, Townsend MF. Posttraumatic Budd-Chiari syndrome treated with thrombolytic therapy and angioplasty. J Trauma 1996;40:294-298.

7. Knudson MM, Collins JA, Goodman SB, McCrory DW. Thromboembolism following multiple trauma. J Trauma 1992;32:2-11.

8. Takeuchi M, Maruyama K, Nakamura M, Chikusa H, Yoshida $\mathrm{T}$, Muneyuki $\mathrm{M}$, et al. Posttraumatic inferior vena caval thrombosis: case report and review of the literature. J Trauma 1995;39: 605-608. 